\title{
Hypoglycemic Coma Due to Insulin Autoimmune Syndrome After Cesarean Section
}

\author{
Zhiyong Yang, Xueyuan Zhong, Enqing Wang, Jing He and Bin $\mathrm{Yi}^{*}$ \\ Department of Anaesthesiology, Southwest Hospital, China
}

Received: 監: December 12, 2018; Published: 业: December 19, 2018

*Corresponding author: Bin Yi, Department of Anaesthesiology, Southwest Hospital, China

\begin{abstract}
Hypoglycemia which is often multifactorial is seldom investigated by anesthesiologists in the operating room because it is easy to reverse, but if not addressed in time, the complications associated with hypoglycemia may rarely be fatal, especially in critically ill patients. Insulin autoimmune syndrome (IAS) is an uncommon cause of spontaneous hypoglycemia which characterized by autoantibodies to insulin in individuals, the sulfhydryl group containing agents may be one inducing factor. In this report, we report a hypoglycemic coma due to insulin autoimmune syndrome induced by propylthiouracil occurring in a pregnancy woman with thyrotoxic heart disease and pre-eclampsia during the early postpartum period after cesarean section with intraspinal anesthesia.
\end{abstract}

Keywords: Hypoglycemic Coma; Thyrotoxic Heart Disease; Insulin Autoimmune Syndrome; Propylthiouracil

Abbreviations: IAS: Insulin Autoimmune Syndrome; FT3: Free Iodothyronine 3; FT4: Free thyroxine 4; TSH: Thyroid Stimulation Hormone; CVP: Central Venous Pressure; HLA: Human Leukocyte Antigen

\section{Introduction}

Hypoglycemia which is often multifactorial is seldom investigated by anesthesiologists in the operating room because it is easy to reverse, but if not addressed in time, the complications associated with hypoglycemia may rarely be fatal, especially in critically ill patients. Insulin autoimmune syndrome (IAS) is an uncommon cause of spontaneous hypoglycemia which characterized by autoantibodies to insulin in individuals, the sulfhydryl group containing agents may be one inducing factor. We report a hypoglycemic coma due to insulin autoimmune syndrome induced by propylthiouracil occurring in a pregnancy woman with thyrotoxic heart disease and pre-eclampsia during the early postpartum period after cesarean section with intraspinal anesthesia.

\section{Case Report}

A 21-year-old woman was transferred to our hospital at 25 weeks of gestation with thyrotoxic heart disease (cardiac function in grade IV), pre-eclampsia, pneumonia, moderate pulmonary hypertension, premature rupture of membranes. On physical examination, she experienced palpitations, anhelation in the sitting position, the heart rate was 130 beats/min and blood pressure were 173/119mmHg. Thyroid function tests showed: free iodothyronine
(FT3 27.31pmol/l), free thyroxine (FT4>100.00pmol/l) and thyroid stimulation hormone (TSH $<0.005 \mathrm{uIU} / \mathrm{ml}$ ). The echocardiography displayed cardiac enlargement, mild pulmonary hypertension with an estimated pulmonary arterial systolic pressure of $61 \mathrm{mmHg}$. Her medical history was significant for Grave's disease and no standard treatment before pregnancy. After multidisciplinary consultation, in consideration of the heart failure and premature rupture of membranes one month, it was decided to deliver the fetus urgently. The day before surgery, propylthiouracil 300mg/day were administrated to control the thyroid hyperactivity, intravenous diuretics 20mg/day and cedilanid (Lanasotside C, also known as Isolanid) $0.8 \mathrm{mg} /$ day were administrated to promote the heart function. The central venous catheters were inserted in the right internal jugular vein for monitoring the central venous pressure (CVP).

In the operating room, the puncture site (L3-4 intervertebral) was identified for employing the combined spinal-epidural anesthesia. when the cerebrospinal fluid was observed, $7.5 \mathrm{mg} 0.5 \%$ hyperbaric bupivacaine was injected into the subarachnoid space slowly and an epidural catheter was placed smoothly. After test dose of $3 \mathrm{~mL} 2 \%$ lidocaine was injected, an additional dose of $5 \mathrm{~mL}$ $2 \%$ lidocaine was re-injected. In the meantime, a radial arterial line 
was established under local anesthesia. When the sensory block level rose to $\mathrm{T} 6$ the surgery commenced. The procedure lasted 97min and went on uneventfully. The Apgar score of newborns was 5-10-10 at 1,3 , and $5 \mathrm{~min}$ after birth. When the patient left the operating room, the arterial blood gases analysis showed $\mathrm{PH}$ 7.36, PCO2 27mmHg and BE $-8.9 \mathrm{mmol} / \mathrm{l}$, Glu $6.4 \mathrm{mmol} / \mathrm{l}$. In the Post-natal Ward, propylthiouracil $150 \mathrm{mg}$ /day and antisterone $20 \mathrm{mg} /$ day were administrated, $120 \mathrm{ml}$ of $5 \%$ dextrose was given every hour and blood glucose was measured every 4 hours. About 22 hours after surgery, the patient complained of palpitations and sweating followed by unconsciousness. Then the $\mathrm{SpO}_{2}$ gradually decreased to $87 \%$ and emergency intubation was performed, an immediately arterial blood gas revealed: $\mathrm{PH} 7.10, \mathrm{PCO}_{2} 58 \mathrm{mmHg}$ and Glu $0.2 \mathrm{mmol} / \mathrm{L}$. $50 \%$ Dextrose $20 \mathrm{ml}$ was administration. Because the serious underlying diseases (pneumonia, thyrotoxic heart disease), the patient was transfered to intensive care unit in the department of cardiology and closely monitored by cardiologist, obstetrist and endocrinologist for 48 hours, the serum total insulin and autoantibodies to insulin were found to be greatly increased (Figure 1).

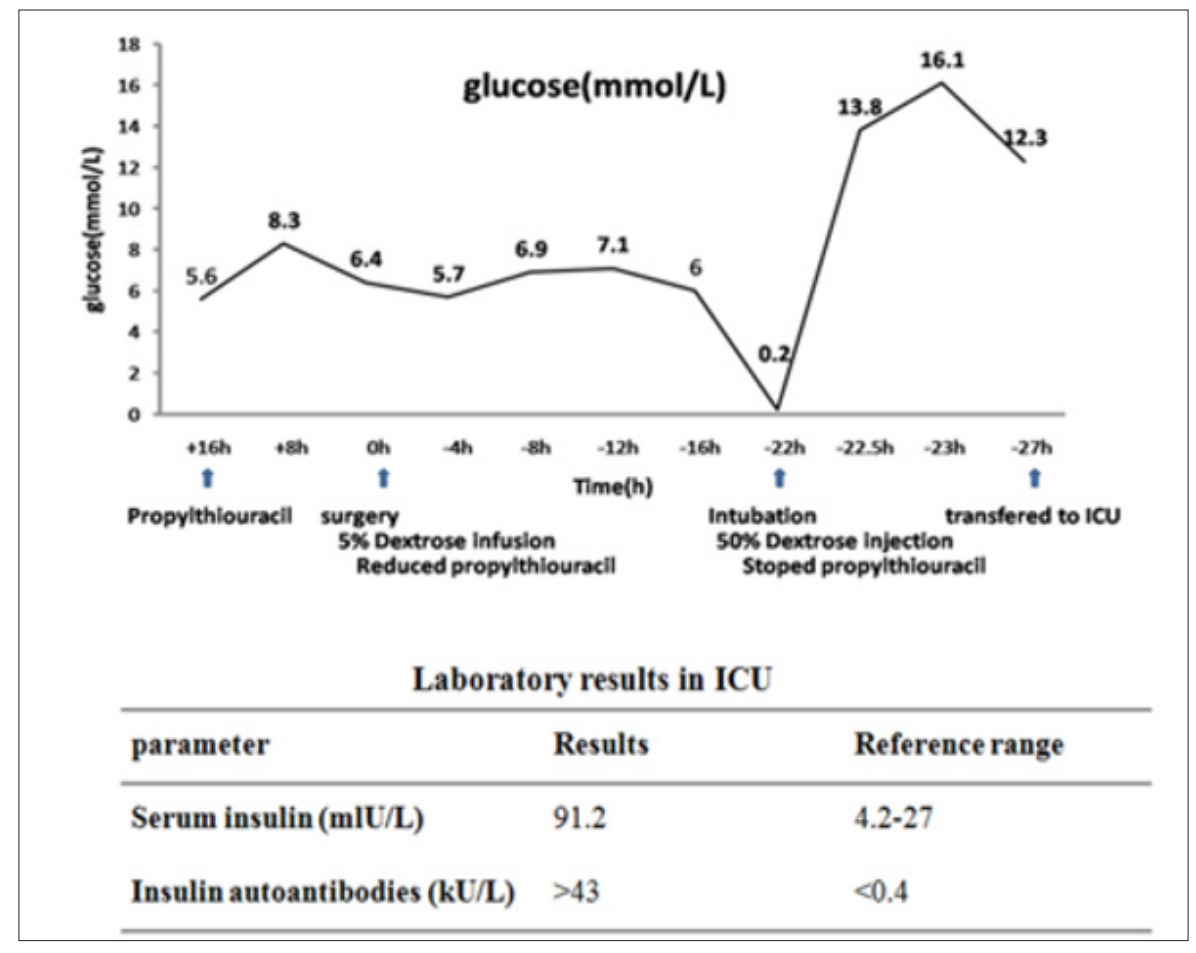

Figure 1: Patient's individual auto-reactivity.

\section{Discussion}

In this case, we found that the unexpected hypoglycemia was the primary cause of the deterioration of the patient's bad situation. Although autoimmune hypoglycemia is a rare cause of hypoglycemia, enhanced clinical suspicion and systematic evaluation should be taken to this patient with autoimmune diseases such as Graves' disease or exposed to sulfa-containing drugs. The mechanism of IAS has not been fully understood, autoimmune disease and drug-induced may related to the pathogenesis of this syndrome [1]. Graves' disease is the most common of autoimmune disorders associated with IAS [2]. Furthermore, IAS seems to be a significantly genetic predispositions, Human Leukocyte Antigen (HLA) types had a strong association with the IAS. HLA- DRB1*0406 as an insulin antigen-presenting major histocompatibility complex molecule has been confirmed to be the highest risk for the susceptibility to IAS [3]. In our case, the patient had on history of antidiabetic and insulinoma, the hypoglycemic state with propylthiouracil use and accompany with Graves' disease, the serum total insulin and autoantibodies to insulin were found to be greatly increased.
Therefore, propylthiouracil-induced IAS were highly considered. In addition, thyrotoxicosis with cardiac failure during pregnancy was associated with a high mortality, the anesthetic management for this parturient undergoing caesarean section is challenging [4]. The perioperative maintenance of hemodynamic stability avoids impairing cardiac function and prevent thyroid storm are crucial for surgical success [5]. We adopted slow incremental cardiostable regional technique (lower dose spinal component and careful staged top up of epidural) to prevent severe hemodynamic fluctuation and provide satisfactory anesthesia. The surgery and anesthesia were successful, the poor condition of pregnancy was improved. Unfortunately, unexpected hypoglycemia made all efforts worthless. Therefore, as the "common" complication among patients with Graves' disease, the IAS should deserve more attention, especially to the patients expose to the medic ion contain sulfhydryl group. The peripartum physicians, including the anesthesiologists, should consider every detail to the improve the outcome of the critically pregnancy. 


\section{Acknowledgement}

Written permission has been taken from the patient's family for publication.

This paper is supported by the Project supported by grants 81870422 from the National Science Foundation of China (NSFC).

\section{References}

1. Censi S, Albergoni MP, Gallo N, Plebani M, Bosaro M, et al. (2017) Insulin autoimmune syndrome (Hirata's disease) in an Italian patient: a case report and review of the literature. Clinical chemistry and laboratory medicine 56(6): 889-895.

2. Savas Erdeve S, Yilmaz Agladioglu S, Onder A, Peltek Kendici HN, Bas VN, et al. (2014) An uncommon cause of hypoglycemia: insulin autoimmune syndrome. Hormone research in paediatrics 82(4): 278-282.

ISSN: 2574-1241

DOI: $10.26717 / B J S T R .2018 .12 .002244$

Bin Yi. Biomed J Sci \& Tech Res

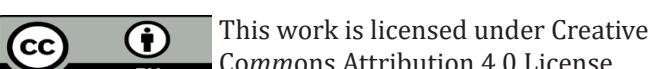

Submission Link: https://biomedres.us/submit-manuscript.php
3. Murakami M, Mizuide M, Kashima K, Kojima A, Tomioka SI, et al. (2000) Identification of monoclonal insulin autoantibodies in insulin autoimmune syndrome associated with HLA-DRB1*0401. Hormone research 54(1): 49-52.

4. Korevaar TIM, Medici M, Visser TJ, Peeters RP (2017) Thyroid disease in pregnancy: new insights in diagnosis and clinical management. Nature reviews Endocrinology 13(10): 610-622.

5. Liao Z, Xiong Y, Luo L (2016) Low-dose spinal-epidural anesthesia for Cesarean section in a parturient with uncontrolled hyperthyroidism and thyrotoxic heart disease. Journal of anesthesia 30(4): 731-734.

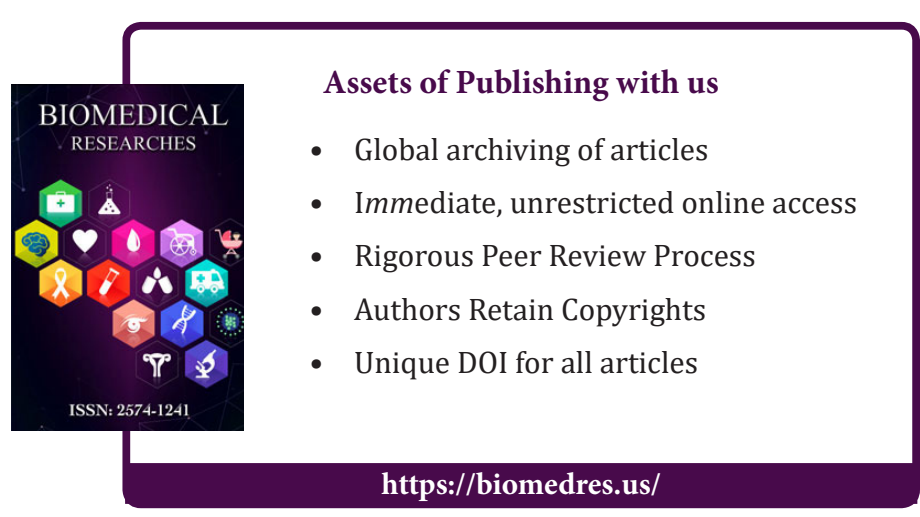

